\title{
Opportunities and Challenges of Integrating Internet of Things (IOT) With Infrastructure of Healthcare Units
}

\author{
CH. M. Shruthi \\ Assistant Professor, Information Technology, \\ Stanley College of Engineering and Technology for Women, Hyderabad, India
}

\begin{abstract}
Internet of Things (IOT) is the technology that can help integrate both physical and digital worlds. With associated technologies like Wireless Sensor Networks (WSN), communication standards and Radio Frequency Identification (RFID), to mention few, it has got plenty of use cases in the real world. One such use case is healthcare industry. Since healthcare industry needs seamless integration and quality in health care services, IoT has become crucial for taking the industry to the next level in providing satisfactory services. There are many benefits of integrating IoT with the infrastructure of healthcare units. One important benefit is real time health monitoring. With wearable technology patients can have body sensors and doctor can view vital signs of patients live. This could help in providing real time healthcare services so as to provide timely diagnosis and treatments. It also avoids many conventional barriers like travelling and wasting time. Still it is in its inception therefore, this paper throws light on the present state-of-theart of IoT and its integration with healthcare units. It provides useful insights on various aspects of integration besides giving recommendations.
\end{abstract}

Keywords: Internet of Things (IOT), Healthcare Industry, Wearable Sensors, Intelligent Systems, Sensor Networks, Communication Standards

\section{INTRODUCTION}

Healthcare is an essential part of life. Unfortunately, the steadily aging population and the related rise in chronic illness is placing significant strain on modern healthcare systems [1], and the demand for resources from hospital beds to doctors and nurses is extremely high [2]. Evidently, a solution is required to reduce the pressure on healthcare systems whilst continuing to provide high-quality care to at-risk patients. The Internet of Things (IoT) has been widely identified as a potential solution to alleviate the pressures on health-care systems, and has thus been the focus of much recent research [3] [4]. A considerable amount of this research looks at monitoring patients with specific conditions, such as diabetes [5] or Parkinson's disease [6]. Further research looks to serve specific purposes, such as aiding rehabilitation through constant monitoring of a patient's progress [4]. Emergency healthcare has also been identified as a possibility by related works [7], [8], but has not yet been widely researched.

Several related works have previously surveyed specific areas and technologies related to IoT healthcare. An extensive survey is presented in [9], with focus placed on commercially available solutions, possible applications, and remaining problems. Each topic is considered separately, rather than as part of an overarching system. In [10], data mining, storage, and analysis are considered, with little mention of integration of these into a system. Sensor types are compared in [11], with some focus placed on communications. However, it is hard to draw an image of a complete system from this paper. Finally, in [8], sensing and big data management is considered, with little regard for the network that will support communications.

This paper therefore makes a unique contribution in that it identifies all key components of an end-to-end Internet of Things healthcare system, and proposes a generic model that could be applied to all IoT-based healthcare systems. This is vital as there are still no known end-to-end systems for remote monitoring of health in the literature. This paper further provides a comprehensive survey of the state-of-the-art technologies that fall within the proposed model. Focus is placed on sensors for monitoring various health parameters, short- and long-range communications standards, and cloud technologies. This paper distinguishes itself from the previous major survey contributions by considering every essential component of an IoT-based healthcare system both separately and as a system. 


\section{International Advanced Research Journal in Science, Engineering and Technology}

Vol. 5, Issue 10, October 2018

\section{HEALTHCARE AND THE INTERNET OF THINGS}

Research in related fields has shown that remote health monitoring is plausible, but perhaps more important are the benefits it could provide in different contexts. Remote health monitoring could be used to monitor non-critical patients at home rather than in hospital, reducing strain on hospital resources such as doctors and beds. It could be used to provide better access to healthcare for those living in rural areas, or to enable elderly people to live independently at home for longer. Essentially, it can improve access to healthcare resources whilst reducing strain on healthcare systems, and can give people better control over their own health at all times. In fact, there are relatively few disadvantages of remote health monitoring. The most significant disadvantages include the security risk that comes with having large amounts of sensitive data stored in a single database, the potential need to regularly have an individual's sensors recalibrated to ensure that they're monitoring accurately, and possible disconnections from healthcare services if the patient was out of cellular range or their devices ran out of battery. Fortunately, these issues are all largely solvable, and are already being addressed in the literature, as will be highlighted throughout the remainder of this paper. As progress continues to be made to reduce the disadvantages, IoT-based systems for remote health monitoring are becoming an increasingly viable solution for the provision of healthcare in the near future.

\section{A. A Model for Future Internet of Things Healthcare Systems}

After reviewing this wide range of existing IoT-based health-care system, several requirements for the design of such systems become apparent. Each of these papers emphasizes the use of sensors for monitoring patient health. All regard wearable sensors, namely wireless and externally-wearable sensors, as essential to their respective systems. Several works [12], [6] also suggest the use of environmental or vision-based sensors around the home. However, this restricts the usefulness of the system to one physical location. It would be preferable to implement all essential sensors as small, portable, and externally wearable nodes. This would provide patients with a non-intrusive and comfortable solution that is capable of monitoring their health wherever they go. This would make patients more receptive to using health monitoring technology than they would be if implantable sensors or cameras were required. Additionally, repairing or replacing externally wearable nodes would be simple when compared to implanted sensors or vision-based sensors installed in the home. Existing systems highlight that communications are also essential for an Internet of Things healthcare system. In several existing system models [5], [6], [13], short-range communications, such as Bluetooth, are suggested for transferring sensor data to a smart phone to be processed. Long-range communications such as LTE can then be used to transfer the processed information from the patient to the healthcare provider, typically a doctor, through SMS or the Internet. The key limitation of this is that smart phones typically have limited battery life, requiring frequent recharging; a patient with a battery would be a patient disconnected from health-care providers. A lowpowered node designed specifically for managing healthcare information would be preferable.

1) Wearable Sensor \& Central Nodes: Wearable sensor nodes are those that measure physiological conditions. Recommended sensors are those that measure the vital signs - pulse, respiratory rate, and body temperature - as these are the essential signs for determination of critical health. Further sensors that could be implemented are blood pressure and blood oxygen sensors, as these parameters are often taken alongside the three vital signs. Special-purpose sensors such as blood-glucose, fall detection, and joint angle sensors could also be implemented for systems targeting a specific condition. The central node receives data from the sensor nodes. It processes this information, may implement some decision making, and then forwards the information to an external location. A dedicated central node would be preferred to a smart phone as battery life could be improved by having only functionality relevant to a healthcare IoT system.

2) Short-Range Communications: For sensors to communicate with the central node, a short-range communications method is required. There are several important requirements to consider when choosing a short-range communications standard, including effects on the human body, security, and latency. The chosen method should have no negative effects on the human body, as any such effects could cause additional health concerns for patients. It should also provide strong security mechanisms to ensure that sensitive patient data cannot be accessed by an attacker. Finally, low-latency is essential for time-critical systems, such as a system that monitors critical health and calls for an ambulance if the need arises. In such systems, time delays could be the difference between life and death. In applications that are not time-critical, low-latency would not need to be prioritized as highly, but is still preferable.

3) Long-Range Communications: Data obtained by the central node is not useful unless some-thing can be done with it. This data should be forwarded to a database where relevant parties (such as caretakers or doctors) can securely access it. There are again several considerations when selecting a suitable long-range communications standard for use in a healthcare system, including security, error correcting capabilities, robustness against interference, low-latency, and high availability. As with short-range communications, strong security is important to ensure that sensitive patient data remains private and cannot be altered or imitated. Low-latency is again important in time-critical applications, 


\section{International Advanced Research Journal in Science, Engineering and Technology}

Vol. 5, Issue 10, October 2018

such as emergency healthcare, where delays in communication could have detrimental effects on patients. High-quality error correcting capabilities and significant robustness against interference are essential, as these ensure that the message sent is the same as the message received. This is important in all healthcare applications, but particularly in emergency situations. Lastly, high availability is essential to ensure that messages will be delivered at all times, regardless of where the patient is physically located. Again, this is of particular importance to time-critical applications, but is preferable for all systems.

4)Secure Cloud Storage Architecture \& Machine Learning: Medical information obtained from patients must be stored securely for continued use. Doctors benefit from knowing a patient's medical history, and machine learning is not effective unless large databases of information are available to it. Based on the literature, cloud storage is the most viable method for storing data. However, providing accessibility for healthcare professionals without compromising security is a key concern [14], [15] that should be addressed by researchers developing healthcare IoT systems. Additionally, machine learning has repeatedly been identified in the literature as a means for improving health-care systems [12], [6], [4], though it has not been widely explored. Machine learning offers the potential to identify trends in medical data that were previously unknown, provide treatment plans and diagnostics, and give recommendations to healthcare professionals that are specific to individual patients. As such, cloud storage architectures should be designed to support the implementation of machine learning on big data sets.

\section{B. Potential Use Cases for the Proposed Model}

The generic model we have proposed for guiding development of future Internet of Things healthcare systems has a number of use cases. To provide context, this subsection discusses several of these use cases, which include aiding rehabilitation, assisting management of chronic conditions, monitoring changes in people with degenerative conditions, and monitoring critical health for the provision of emergency healthcare.

Following our proposed model, a rehabilitation system for knee injuries could be developed by using wearable accelerometer sensors on either side of the knee, to allow for the position and angle of the knee to be calculated. These measurements could be recorded during several activities, such as normal walking and rehabilitation exercises. They could be communicated via short-range communications to a comfortable, wrist-wearable central node, which could then forward information to the cloud via long-range communications. In the cloud, a record of the patient's progress will continue to expand with each received message. Machine learning algorithms could be implemented to identify the patient's progress, predict when they will be fully rehabilitated, and determine whether any exercises are working better than others. This system could easily be adapted for other or additional injuries by modifying which wearable sensors are used. Our model could also be used to develop a system capable of assisting with the management of chronic conditions such as hypertension. Blood pressure could be monitored at several locations on the body at set intervals throughout the day and communicated to the cloud via a wrist-worn central node. Again, a comprehensive record of the patient's blood pressure could be built and machine learning could be used to identify trends such as when the patient's blood pressure is highest. This information could also be used to determine optimal times for the patient to take any medication that they may require to manage their condition, and remind the patient of that using a buzzer or alarm on the central node.

\section{WEARABLE HEALTHCARE SYSTEMS3}

WBANs have been identified as a key component of a healthcare system founded on Internet of Things technology, and as such the development of accurate sensors with low form factor are essential for the successful development of such a system. In this article, we focus on sensors that are non-obtrusive and non-invasive; we exclude sensors such as implantable. Considered are five fundamental sensors - three for monitoring the vital signs of pulse, respiratory rate, and body temperature, and a further two for monitoring blood pressure and blood oxygen, both commonly recorded in a hospital environment?

A. Pulse Sensors: Perhaps the most commonly read vital sign, pulse can be used to detect a wide range of emergency conditions, such as cardiac arrest, pulmonary embolisms, and vasovagal syncope. Pulse sensors have been widely researched, both for medical purposes and for fitness tracking. Pulse can be read from the chest, wrist, earlobe, fingertip, and more. Earlobe and fingertip readings provide high accuracy, but are not highly wearable. A chest-worn system is wearable, but wrist sensors are generally considered most comfortable for a long-term wearable system [16]. Commercially, several fitness tracking chest straps and wrist watches are available with pulse measurement functionality. These include HRM-Tri by Garmin [17], H7 by Polar [18], FitBit PurePulse [19], and TomTom Spark Cardio [20]. However, these companies all disclose that their devices are not for medical use and should not be relied upon for detecting health conditions. As such, the sensing systems employed by these devices cannot be directly implemented into a critical health monitoring system. Much research has been conducted into suitable methods for sensing pulse. Sensor types developed, used, and analyzed in recent works include pressure, Photo Plethysmo Graphic 


\title{
International Advanced Research Journal in Science, Engineering and Technology
}

\author{
Vol. 5, Issue 10, October 2018
}

(PPG), ultrasonic, and radio frequency (RF) sensors. PPG sensors operate by an LED transmitting light into the artery, with a photodiode receiving the amount not absorbed by the blood, as shown in Figure 1. Changes in the amount of light can be recorded and a pulse rate can thus be determined. Pressure sensors aim to mimic a healthcare professional manually reading the radial pulse by pressing down with their fingers. As shown in Figure 2, the sensor is placed firmly against the wrist, and pressure is continuously measured to acquire a pulse waveform.

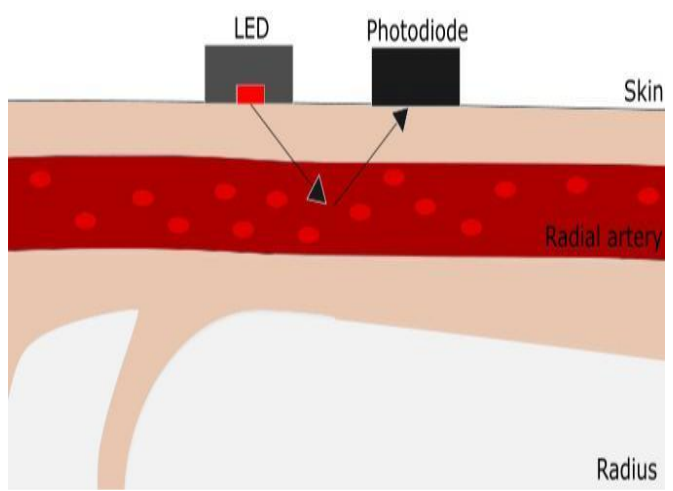

Figure 1. Photoplethysmographic pulse sensor.

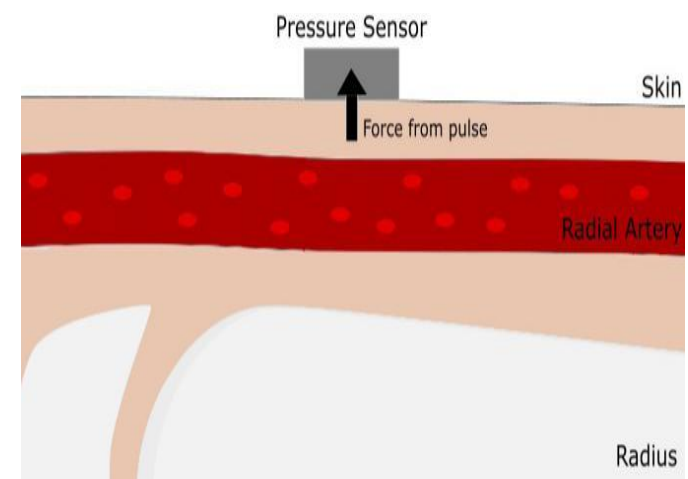

Figure 2. Pressure-based pulse sensor.

In [21], a flexible and highly-sensitive pressure sensor for pulse detection is developed and tested, showing promising results. However, increasing the sensitivity to better detect pulse also increases the amount of noise that is detected due to movement of the wearer. This sensor was tested in at-rest conditions, and further research would be required to determine that it performed well during motion. Pressure sensors and PPG sensors are combined in [22] and [23], where pulse sensor modules are developed with arrays of nine PPG sensors and one pressure sensor. Pulse is taken from multiple points on the wrist, providing clear pulse readings and the potential to use these readings for diagnostics of certain diseases such as diabetes.

B. Respiratory Rate Sensors: Another of the vital signs is respiratory rate, or the number of breaths a patient takes per minute. Monitoring respiration could aid in the identification of conditions such as asthma attacks, hyperventilation due to panic attacks, apnea episodes, lung cancer, obstructions of the airway, tuberculosis, and more. Due to the importance of respiration, many previous works have developed sensors for measuring respiratory rate. In inspecting the previous works, several types of respiratory rate sensor emerge. The first is a nasal sensor based on a thermistor, as is used in [24]. The principle that these sensors are based on is that air exhaled is warmer than the ambient temperature. As such, the sensor uses the rise and fall of temperature to count the number of breaths taken. This is shown to work reasonably well, but accuracy may be compromised by other sources of temperature fluctuations - for example if worn by a chef working in a kitchen. It is also not highly wearable, as it is obstructive and easily noticeable. Echocardiogram (ECG) signals can also be used to obtain respiration rate. This is called ECG Derived Respiration (EDR), and is used in [25] to determine respiration patterns and detect apnea events. This method reads respiratory rate reasonably well, but is again limited by the wear-ability. ECG contacts are uncomfortable and would likely cause irritation to the skin if used continuously. Additionally, ECG contacts are not reusable and would need to be regularly replaced.

C. Body Temperature Sensors: The third vital sign is body temperature, which can be used to detect hypothermia, heat stroke, fevers, and more. As such, body temperature is a useful diagnostics tool that should be included in a wearable healthcare system. Recent works surrounding the measurement of body temperature all use thermistor-type 


\title{
International Advanced Research Journal in Science, Engineering and Technology
}

\author{
Vol. 5, Issue 10, October 2018
}

sensors. In [26] and [27], the common negative-temperature-coefficient (NTC) type temperature sensors were used, while positive-temperature-coefficient (PTC) sensors were considered in [28] and [29]. In all studies, the thermostats were shown to measure a suitable range of temperatures for monitoring the human body, with acceptable levels of error. Therefore, it is strongly recommended that these sensor types continue to be used by future system designers.

D. Blood Pressure: Whilst not a vital signs itself, blood pressure (BP) is frequently measured alongside the three vital signs. Hyperten-sion (high BP) is a known risk factor for cardiovascular disease, including heart attack. It is also one of the most common chronic illnesses, affecting 32\% of adult Australians. Of those affected, $68 \%$ had uncontrolled or unmanaged hypertension [30]. As such, incorporating BP into a WBAN for healthcare would provide vital information for many patients. Nonetheless, designing a wearable sensor for continuously and non-invasively monitoring blood pressure remains a challenge in the field of healthcare IoT. A significant number of works [31] [32] have attempted to obtain an accurate estimate of BP through calculation of pulse transit time (PTT) - the time taken between pulse at the heart and pulse at another location, such as the earlobe or radial artery. Another work endeavored to measure this property between the ear and wrist [33], while another looked to calculate it between the palm and the fingertip of a hand [34]. PTT is known to be inversely proportional to systolic blood pressure (SBP), and is typically determined using an electrocardiogram on the chest and a PPG sensor on the ear, wrist, or alternate location.

E. Pulse Oximetry Sensors: Pulse oximetry measures the level of oxygen in the blood. Like blood pressure, blood oxygen level is not a vital sign, but does serve as an indicator of respiratory function and can aid in diagnostics of conditions such as hypoxia (low oxygen reaching the body's tissues). As such, pulse oximetry is a valuable addition to a general health monitoring system. Pulse oximeters measure blood oxygen by obtaining PPG signals. Usually, two LEDs - one red, one infrared - are directed through the skin. Much of this light is absorbed by the hemoglobin in the blood, but not all. The amount of light not absorbed is measured by receiving photodiodes, and the difference between the received lights is used to calculate blood oxygen. As highlighted in Figure 3, LED lights can either be passed through an appendage (normally a finger) to a photodiode on the opposite side, or can be directed at an angle so that some light reflects to a photodiode on the same side of the appendage. These are called absorbance-mode and reflectance-mode PPG sensors respectively.
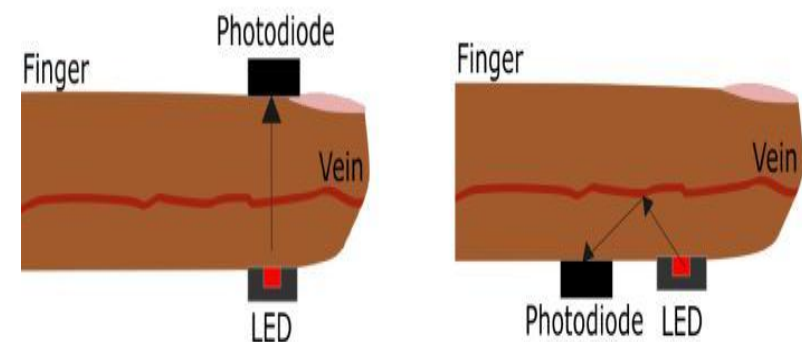

Figure 3. Absorbance-mode vs. reflective-mode PPG sensors for pulse oximetry.

Classically, pulse oximeters are worn as a finger clip wired to a medical monitor. Several recent works have attempted to make more portable devices. In [35], a low-power pulse oximeter is designed with the aim of improving wear ability. Two techniques are used to reduce power consumption. The first - named "minimum SNR tracking" - continuously calculates the current signal-to-noise-ratio (SNR) and adjusts the length of time that the LED is in the "on" state for accordingly - the higher the SNR is, the longer that the LED needs to be on to gain accurate readings. The second, named "PLL tracking", estimates when the peaks and troughs of the PPG signal are likely to occur, and samples only at these times to acquire this important information. Up to 6x less power was consumed through implementing both techniques, and the worst error recorded was a $2 \%$ difference between actual and measured blood oxygen levels. This is a significant contribution towards making pulse oximeters more wearable, but reductions in the level of error are desirable.

F. Other Wearable Sensors For Healthcare: Aside from the sensors that measure critical health parameters, there are several special-purpose wearable sensors that may be useful in systems focused on monitoring a specific condition. Echocardiograms (ECGs) can be used to evaluate heart health, and several wearable sensors have been developed to acquire these signals. In [36], an armband-based ECG sensor was developed and measures with reasonable accuracy. ECG sensors have also successfully been developed for integration in helmets [37] and more traditional chest-straps [38]. The helmet in [37] also features an electroencephalogram (EEG) sensor. EEGs measure brain activity, and could generally be used to monitor seizures, sleep disorders, and progress after a head injury. Other EEG systems have been developed for specific purposes, such as for detecting driver drowsiness [39] or stress management [40]. Both systems measure EEG through a relatively wearable headband. 


\title{
International Advanced Research Journal in Science, Engineering and Technology
}

\author{
Vol. 5, Issue 10, October 2018
}

\section{COMMUNICATIONS STANDARDS}

Communications related to Internet of Things for healthcare can be classified into two main categories: short-range communications, and long-range communications. The former is used to communicate between devices within the WBAN, whilst the latter provides connection between the central node of the WBAN and a base station (such as a healthcare provider). In this paper, both types of communications are considered with equal importance.

A. Short-Range Communications: In the context of wearable healthcare systems, short-range communications are often used between nodes, particularly between sensor nodes and the central node where data processing occurs. Although short-range communications standards can be used for other purposes (i.e. developing mesh networks for smart lighting), this survey focuses on the purpose of developing a small WBAN that is comprised of only a few sensors and a single central node. Many short-range communications standards exist, but perhaps the most commonly used ones in IoT are Bluetooth Low Energy (BLE) and ZigBee. The key features of these two standards are highlighted in Table 1, and this section further analyzes these standards and considers their suitability for implementation into an IoT healthcare system.

B. Long-Range Communications: Low-Power Wide-Area Networks (LPWANs) are a subset of long-range communications standards with high suitability for IoT applications. The range of a LPWAN is generally several kilometers, even in an urban environment. This is significantly longer than the range of traditional IoT communications types such as WiFi or Bluetooth, whose ranges are in the order of meters and thus would require extensive and costly mesh networking or similar to be plausible for healthcare.

\section{CLOUD-BASED IOT HEALTHCARE SYSTEMS}

Cloud technologies have been widely researched due to their usefulness in big data management, processing and analytics. Several related works have surveyed the literature on using cloud technologies for IoT purposes such as smart grid [41] and mobile cloud computing for smart-phones [42], [43], where complex computations are of loaded from low-resource mobile devices to the high-power environment of the cloud, before the result is returned to the mobile device. These works consider data storage and data processing as key advantages of cloud technologies.

Further related works have reviewed the state of cloud-centric healthcare. The use of cloud technology for health record storage is considered in [44], which also overviews cloud technologies as a complete field. Storage is considered further [10] and [45], with particular focus on how a large database could be used for data analysis and trend determination. While each of these related works provides valuable insight into the field of cloud technologies, there is no known article that considers all advantages, disadvantages, challenges, and opportunities that cloud presents to healthcare systems based on WBANs and the IoT. In this section, we bridge the gap in the literature by presenting recent works regarding cloud-centric healthcare, analyzing key challenges, and providing recommendations for future research directions.

A. Data Processing and Analytics: There are several types of data processing that can be per-formed using cloud technologies, but the most relevant are computational of loading and machine learning. Computational of loading involves using the cloud to perform complex data processing beyond the capabilities of low-resource wear-able devices. By sending raw or partially processed sensor data to the cloud, the computing resources of many machines can be utilized for processing. Using this high-powered computing environment over processing on the standalone mobile device offers many advantages; more complex algorithms can be executed, results can be obtained significantly faster and battery life will be extended in mobile devices due to less processing occurring internally.

Complicated sensor nodes such as those measuring ECG data, blood pressure, or accelerometers for fall detection would benefit greatly from computational of loading. For example, ECGs have a standard shape, and different deviations from this shape can indicate several different heart problems including arrhythmia, heart in ammation, and even cardiac arrest. A small, low-powered sensor node could not analyze ECG readings rapidly using machine learning algorithms to determine the patient's state of health. However, if the raw data was offloaded to cloud, high-power processing could be performed to determine the shape of the ECG before machine learning algorithms compare it to the characteristic shape, identify any serious differences between the shapes, and determine what condition is causing them.

B. Security and Privacy in the Cloud: Security remains a key issue in cloud-based systems. In a healthcare environment, it is essential that a patient's health information is readily accessible to authorized parties including doctors, nurses, specialists, and emergency services. It is also essential that the patient's sensitive health data is kept private. If malicious attacks revealed the patient's health data, it could have many negative ramifications for the patient, including exposing them to identity theft or making it dif cult for them to obtain insurance. Worse still, if the malicious 


\title{
International Advanced Research Journal in Science, Engineering and Technology
}

\author{
Vol. 5, Issue 10, October 2018
}

attacker altered a patient's health record, it could have detrimental effects on the patient's health. Access control policies and data encryption are two means of securing cloud-centric healthcare systems. An access control policy specifies who is authorized access to the patient's health data, and how much access they are allowed. It would also implement an authentication mechanism (e.g. password, facial recognition, etc.) that verifies the identity of the party attempting to access the data. Meanwhile, data encryption provides security for the data whilst in data storage. Strong data encryption would prevent an attacker from reading sensitive health information, even if they did gain access to the database.

\section{FINDINGS AND RECOMMENDATIONS}

The lessons learned through conducting this survey highlight several areas for further research. In terms of sensors, much progress has been made but there are still no available devices that match the accuracy of hospital-grade devices without compromising energy efficiency or wearability. This is especially true of complex devices such as blood pressure and respiratory rate sensors, both of which would be invaluable to the field of medicine. As such, further research efforts should be made towards improving the quality of these sensors until they are highly accurate, reliable, and comfortably wearable. In our own future works, we will be placing particular focus on developing a blood pressure monitor that is more wearable than the works presented in this paper, without compromising accuracy. We will also look at reducing the impact of motion on sensors, particularly for respiratory rate and pulse sensors.

There is still much room for improvement in security and privacy for cloud-based healthcare. No known encryption scheme is ideal for protecting data whilst providing accessibility for authorized parties and enabling machine learning. $\mathrm{ABE}$ and FHE are schemes that provide appealing characteristics, but are not lightweight enough for implementation into wearable devices. Improving these schemes is the first active area of research. Upon improving the schemes individually, a lightweight ABE-FHE hybrid scheme should be considered, as it could potentially provide all the desirable characteristics for cloud-based healthcare security.

Overall, there is no known end-to-end system for general or specific purposes that contains all components in our proposed model; wearable sensors, short- and long-range communications, cloud-based storage, and machine learning. Developing such a system would be a significant achievement in the field of IoT-based healthcare, and should be considered as the ultimate goal for researchers in this area. In our own future works, we will be striving to reach this goal through the development of a wearable, IoT-based system for the provision of emergency healthcare that incorporates health sign monitoring, machine learning for diagnostics, and long-range communications via LPWANs to notify emergency service providers when a patient needs urgent help.

\section{CONCLUSION AND FUTURE WORK}

In this paper we reviewed the present state of the art of IoT integrated healthcare system including the technologies behind it, wearable sensors, standards, opportunities and implications. Many wearable devices that can be used to have real time health monitoring with IoT connected to healthcare infrastructure. They are used to measure oxygen levels in blood, blood pressure and other vital signs. Communication standards with short range and long rage are discussed. The possible usage of cloud computing is explored. Since IoT generates huge amount of data, cloud storage and data analytics are essential for successful implementation of IoT with healthcare unit. The security risks with cloud are also covered. Encryption and access control policies with respect to safeguarding healthcare units integrated with IoT is discussed. From the research it is understood that Healthcare industry benefits with IoT integration. In the same fashion, it can be used with many industries in the real world. In future we investigate the suitability of IoT integration with other industries.

\section{REFERENCES}

[1]. Australian Institute of Health and Welfare. (2014). Australia's Health. [Online]. Available: http://www.aihw.gov.au/WorkArea/ DownloadAsset.aspx-?id=60129548150

[2]. E. Perrier, Positive Disruption: Healthcare, Ageing and Participation in the Age of Technology. Sydney, NSW, Australia: The McKell Institute, 2015.

[3]. P. Gope and T. Hwang, “BSN-care: A secure IoT-based modern health-care system using body sensor network," IEEE Sensors J., vol. 16, no. 5,1368 1376, Mar. 2016.

[4]. Y. J. Fan, Y. H. Yin, L. D. Xu, Y. Zeng, and F. Wu, “'IoT-based smart rehabilitation system," IEEE Trans. Ind. Informat., vol. 10, no. 2, 1568 1577, May 2014

[5]. S.-H. Chang, R.-D. Chiang, S.-J. Wu, and W.-T. Chang, “A context-aware, interactive M-health system for diabetics," IT Prof., vol. 18, no. 3,14 22, May/Jun. 2016.

[6]. C. F. Pasluosta, H. Gassner, J. Winkler, J. Klucken, and B. M. Esko er, “An emerging era in the management of Parkinson's disease: Wearable technologies and the Internet of Things," IEEE J. Biomed. Health Inform., vol. 19, no. 6, pp. 1873 1881, Nov. 2015.

[7]. S. Sarkar and S. Misra, "From micro to nano: The evolution of wire-less sensor-based health care," IEEE Pulse, vol. 7, no. 1, pp. 21 25, Jan./Feb. 2016

[8]. Y. Yin, Y. Zeng, X. Chen, and Y. Fan, “The Internet of Things in healthcare: An overview," J. Ind. Inf. Integr., vol. 1, pp. 3 13, Mar. 2016. [Online]. Available: http://www.sciencedirect.com/science/ article/pii/S2452414X16000066

[9]. S. M. R. Islam, D. Kwak, H. Kabir, M. Hossain, and K.-S. Kwak, “'The Internet of Things for health care: A comprehensive survey," IEEE Access, vol. 3, pp. 678 708, 2015. 


\title{
International Advanced Research Journal in Science, Engineering and Technology
}

\author{
Vol. 5, Issue 10, October 2018
}

[10]. D. V. Dimitrov, "Medical Internet of Things and big data in healthcare," Healthcare Inform. Res., vol. 22, no. 3, pp. 156163 , Jul. 2016. [Online]. Available: http://www.ncbi.nlm.nih.gov/pmc/articles/PMC4981575/

[11]. C. C. Y. Poon, B. P. L. Lo, M. R. Yuce, A. Alomainy, and Y. Hao, "Body sensor networks: In the era of big data and beyond," IEEE Rev. Biomed. Eng., vol. 8, pp. 4 16, 2015.

[12]. N. Zhu et al., "Bridging e-health and the Internet of Things: The SPHERE project," IEEE Intell. Syst., vol. 30, no. 4, pp. 39 46, Jul./Aug. 2015.

[13]. G. Wolgast, C. Ehrenborg, A. Israelsson, J. Helander, E. Johansson, and H. Manefjord, Wireless body area network for heart attack detec-tion [education corner]," IEEE Antennas Propag. Mag., vol. 58, no. 5,84 92, Oct. 2016.

[14]. I. Olaronke and O. Oluwaseun, "Big data in healthcare: Prospects, chal-lenges and resolutions," in Proc. Future Technol. Conf. (FTC), Dec. 2016,pp.1152 1157.

[15]. J. Zhou, Z. Cao, X. Dong, and A. V. Vasilakos, "Security and privacy for cloud-based IoT: Challenges," IEEE Commun. Mag., vol. 55, no. 1,pp.26 33, Jan. 2017.

[16]. J. šenko, M. Kos, and I. Kramberger, "Pulse rate variability and blood oxidation content identi cation using miniature wearable wrist device," in Proc. Int. Conf. Syst., Signals Image Process. (IWSSIP), May 2016,pp.1-4.

[17]. Garmin. (2017). HSM-Tri. [Online]. Available: https://buy.garmin.com/ en-AU/AU/p/136403

[18]. Polar. (2017). H7 Heart Rate Sensor. [Online]. Available: https://www. polar.com/au-en/products/accessories/H7_heart_rate_sensor

[19]. FitBit. (2017). FitBit PurePulse. [Online]. Available: https://www. tbit. com/au/purepulse

[20]. TomTom. (2017). TomTom Spark Cardio. [Online]. Available: https:// www.tomtom.com/en_au/sports/ fitness-watches/gps-watch-cardiospark/black-large/

[21]. Y. Shu, C. Li, Z. Wang, W. Mi, Y. Li, and T.-L. Ren, “A pressure sensing system for heart rate monitoring with polymer-based pressure sensors and an anti-interference post processing circuit," Sensors, vol. 15, no. 2,pp.3224 3235, 2015.

[22]. D. Wang, D. Zhang, and G. Lu, “'A novel multichannel wrist pulse system with different sensor arrays," IEEE Trans. Instrum. Meas., vol. 64, no. 7,pp.2020 2034, Jul. 2015

[23]. D. Wang, D. Zhang, and G. Lu, “An optimal pulse system design by multichannel sensors fusion," IEEE J. Biomed. Health Informat., vol. 20, no. 2, pp. 450459 , Mar. 2016

[24]. S. Milici, J. Lorenzo, A. Lázaro, R. Villarino, and D. Girbau, "Wireless breathing sensor based on wearable modulated frequency selective surface," IEEE Sensors J., vol. 17, no. 5, pp. 1285 1292, Mar. 2017.

[25]. C. Varon, A. Caicedo, D. Testelmans, B. Buyse, and S. van Huffel, “A novel algorithm for the automatic detection of sleep apnea from singlelead ECG," IEEE Trans. Biomed. Eng., vol. 62, no. 9,pp.2269 2278, Sep. 2015.

[26]. P. Aqueveque, C. Gutiérrez, F. S. Rodríguez, E. J. Pino, A. Morales, and P. Wiechmann, “Monitoring physiological variables of mining workers at high altitude," IEEE Trans. Ind. Appl., vol. 53, no. 3, pp. 2628 2634, May/Jun. 2017.

[27]. P. Narczyk, K. Siwiec, and W. A. Pleskacz, "Precision human body temperature measurement based on thermistor sensor," in Proc. IEEE 19th Int. Symp. Design Diagnostics Electron. Circuits Syst. (DDECS), Apr. 2016, pp. 1-5.

[28]. T. Nakamura et al., "Development of exible and wide-range polymer-based temperature sensor for human bodies," in Proc. IEEE-EMBS Int. Conf. Biomed. Health Inform. (BHI), Feb. 2016, pp. 485488.

[29]. A. Eshkeiti et al., “A novel self-supported printed exible strain sensor for monitoring body movement and temperature," in Proc. IEEE SENSORS, Nov. 2014, pp. 16151618

[30]. Heart Foundation. (2017). High Blood Pressure Statistics. [Online]. Available: www.heartfoundation.org.au/about-us/what-we-do/heartdisease-in-australia/high-blood-pressure-statistics

[31]. S. S. Thomas, V. Nathan, C. Zong, K. Soundarapandian, X. Shi, and Jafari, “BioWatch: A noninvasive wrist-based blood pressure monitor that incorporates training techniques for posture and subject variabil-ity," IEEE J. Biomed. Health Inform., vol. 20, no. 5, pp. 1291-1300, Sep. 2016.

[32]. D. Griggs et al., "Design and development of continuous cuff-less blood pressure monitoring devices," in Proc. IEEE SENSORS, Oct./Nov. 2016, pp. 1-3.

[33]. Y. Zhang, M. Berthelot, and B. P. Lo, "Wireless wearable photoplethys-mography sensors for continuous blood pressure monitoring," in Proc. IEEE Wireless Health (WH), Oct. 2016, pp. 1-8.

[34]. J. Wannenburg and R. Malekian, “Body sensor network for mobile health monitoring, a diagnosis and anticipating system," IEEE Sensors J., vol. 15, no. 12, pp. $6839-6852$, Dec. 2015.

[35]. S. V. Gubbi and B. Amrutur, "Adaptive pulse width control and sampling for low power pulse oximetry," IEEE Trans. Biomed. Circuits Syst., vol. 9, no. 2, pp. 272-283, Apr. 2015.

[36]. V. P. Rachim and W.-Y. Chung, “'Wearable noncontact armband for mobile ECG monitoring system," IEEE Trans. Biomed. Circuits Syst., vol. 10, no. 6, pp. 11121118 , Dec. 2016.

[37]. W. Von Rosenberg, T. Chanwimalueang, V. Goverdovsky, D. Looney,Sharp, and D. P. Mandic, Smart helmet: Wearable multichannel ECG and EEG," IEEE J. Transl. Eng. Health Med., vol. 4, 2016, Art. no. 2700111.

[38]. E. Spanò, S. D. Pascoli, and G. Iannaccone, "Low-power wearable ECG monitoring system for multiple-patient remote monitoring," IEEE Sen-sors J., vol. 16, no. 13, pp. 5452 5462, May 2016.

[39]. ]G. Li, B.-L. Lee, and W.-Y. Chung, "Smartwatch-based wearable EEG system for driver drowsiness detection," IEEE Sensors J., vol. 15, no. 12, pp. 7169 7180, Dec. 2015

[40]. U. Ha et al., "A wearable EEG-HEG-HRV multimodal system with simultaneous monitoring of tES for mental health management," IEEE Trans. Biomed. Circuits Syst., vol. 9, no. 6, pp. 758 766, Dec. 2015.

[41]. S. Bera, S. Misra, and J. J. P. C. Rodrigues, "Cloud computing appli-cations for smart grid: A survey," IEEE Trans. Parallel Distrib. Syst., vol. 26, no. 5, pp. 1477 1494, May 2015.

[42]. P. Mach and Z. Becvar, "Mobile edge computing: A survey on architec-ture and computation of oading," IEEE Commun. Surveys Tuts., vol. 19, no. 3, pp. 16281656 , 3rd Quart., 2017.

[43]. A. R. Khan, M. Othman, S. A. Madani, and S. U. Khan, “A survey of mobile cloud computing application models," IEEE Commun. Surveys Tuts., vol. 16, no. 1, pp. 393 413, Feb. 2014

[44]. N. Sultan, "Making use of cloud computing for healthcare provision: Opportunities and challenges," Int. J. Inf. Manage., vol. 34, no. 2,pp.177 184, Apr. 2014. [Online]. Available: http://www.sciencedirect. com/science/article/pii/S0268401213001680

[45]. L. A. Tawalbeh, R. Mehmood, E. Benkhlifa, and H. Song, "Mobile cloud computing model and big data analysis for healthcare applications," IEEE Access, vol. 4, pp. 61716180 